Pacific Journal of Mathematic 


\title{
THE FIVE-POINT DIFFERENCE EQUATION WITH PERIODIC COEFFICIENTS
}

\author{
TOMLINSON FORT
}

The five-point difference equation described in $\S 1$ has most of the important second order partial difference equations as special cases and as limiting forms of these the more important partial differential equations of the second order. In the present paper all coefficients are assumed periodic in the same one of the two independent variables. The purpose of the paper is the study of the form of the general solution as affected by the periodic character of the coefficients. This study centers around the roots of the characteristic equation and so-called semi-periodic solutions. The reader is referred to the theorem of $\S 5$ for a precise statement of results.

1. General discussion. Let us be given the five-point equation

$$
\begin{aligned}
k_{1}(i, j) y(i-1, j) & +k_{2}(i, j) y(i+1, j)+k_{3}(i, j) y(i, j-1) \\
& +k_{4}(i, j) y(i, j+1)+k_{5}(i, j) y(i, j)=0
\end{aligned}
$$

where $k_{1}, k_{2}, k_{3}, k_{4}$ and $k_{5}$ are defined for integral values of $i$ and $j$ over the rectangle $1 \leqq i \leqq n \omega-1,1 \leqq j \leqq \omega-1$ where $\mathrm{n}>1$ and $\omega>1$ are integers. This rectangle will be called the defining rectangle and will be denoted by $R$. We assume moreover that

$$
k_{\nu}(i+\omega, j)=k_{\nu}(i, j), \quad \nu=1,2,3,4,5
$$

and that neither, $k_{1}, k_{2}, k_{3}$, nor $k_{1}$ is zero at any point of $R$.

A solution of (1) is a function of $(i, j)$ defined at points of $R$ and at the border points $(i=0, j=1,2, \cdots, \omega-1),(i=n \omega, j=1,2, \cdots, \omega-1)$, $(j=0, i=1,2, \cdots, n \omega-1),(j=\omega . i=1,2, \cdots, n \omega-1)$ and which satisfies (1) at all points of $R$. Notice that this second set of points, namely $R$ plus the border points, form a lattice which is rectangular except that its corner points are missing. It will be referred to as the rectangle $S$.

A fundamental domain is a set of points of $S$ such that there exists one and only one solution taking on prescribed arbitrary values at each point of the set.

All fundamental domains ${ }^{1}$ contain the same number of points. We denote this number by $L$. For the rectangle $S$

Received December 6, 1956. This paper was sponsored by the Office of Ordnance Research of the U. S. Army.

${ }^{3}$ For a detailed discussion see T. Fort, Amer. Math. Monthly, 62, (1955), 161. 


$$
L=2 \omega(n+1)-4 .
$$

We note that all sets of $L$ points belonging to $S$ do not form a fundamental domain.

A fundamental system of solutions consists of $L$ solutions which are linearly independent over a fundamental domain.

If $y_{1}(i, j), y_{2}(i, j), \cdots, y_{L}(i, j)$ are a fundamental system of solutions then any solution $y(i, j)$ can be written

$$
y(i, j)=\sum_{\mu=0}^{L} c_{\mu} y_{\mu}(i, j)
$$

where the $c$ 's are constants.

We choose the following fundamental domain for $S$ namely points where

$$
\begin{aligned}
& j=0, \quad i=1,2, \cdots, n \omega-1 ; \\
& j=1, \quad i=0, \omega, \omega+1, \cdots, 2 \omega-1,2 \omega, 3 \omega, \cdots, n \omega ; \\
& j=2, \quad i=0, \omega, 2 \omega, \cdots, n \omega ; \\
& \cdot . \cdot . \cdot \cdot \cdot \cdot \cdot \cdot . \cdot \\
& j=\omega-1, \quad i=0, \omega, 2 \omega, \cdots, n \omega ; \\
& j=\omega, \quad i=\omega, 2 \omega, \cdots,(n-1) \omega .
\end{aligned}
$$

We shall refer to this particular fundamental domain as $D$. The domain $D$ is pictured in figure 1 with $\omega=4, n=3$. The points of $D$ have no accompanying numeral.

To prove $D$ a fundamental domain simply assign values for $y$ at each of the points of $D$ and fill in the rectangle by means of (1). This can be done in a variety of ways. For example, the value of $y$ at the points of $S$ not in $D$ can be determined in the order indicated by the accompanying numerals in Figure 1.

$\begin{array}{lllllllllll}\dot{2} 1 & 20 & 19 & \cdot & \dot{7} & \dot{8} & \dot{9} & \cdot & \dot{3} 1 & \dot{3} 2 & \dot{3} 3 \\ \text { i8 } & \text { i7 } & \text { i2 } & \cdot & \dot{4} & \dot{5} & \dot{6} & \cdot & \dot{2} 4 & \dot{2} 9 & \dot{3} 0 \\ \text { i6 } & \text { i4 } & \text { i1 } & \cdot & \dot{1} & \dot{2} & \dot{3} & \cdot & \dot{2} 3 & \dot{2} 6 & \dot{2} 8 \\ \text { i5 } & \text { i3 } & \text { i0 } & & \cdot & \cdot & \cdot & \cdot & \dot{2} 2 & \dot{2} 5 & \dot{2} 7\end{array}$

Figure 1.

We now define all coefficients to the right of $i=n \omega-1$ by formula (2). We then define every solution $y(i, j)$ at the points $(n \omega, 0),(n \omega+1,0)$, $\cdots,((n+1) \omega-1,0)$ by the formula 


$$
y(n \omega+\nu, 0)=y(\nu+1,0), \quad \nu=0,1, \cdots, \omega-1 \text {; }
$$

and the points $((n+1) \omega, j), j=1,2, \cdots, \omega-1$ by the formula

$$
y((n+1) \omega, j)=y(0, j) \quad \text { also } \quad y(n \omega, \omega)=y(\omega, \omega) .
$$

This definition serves to determine $y$ over a longer rectangle than $S, n \omega$ being replaced by $(n+1) \omega$, the rectangles being in every other way the same. We call this the rectangle $T$.

BASIC TheOREM. If $y(i, j)$ is a solution over $S$ then $y(i+\omega, j)$ is also a solution over $S$.

This theorem follows immediately from the periodic character of the coefficients in (1).

THEOREM. If $y_{1}(i, j), y_{2}(i, j), \cdots, y_{L}(i, j)$ are a fundamental system of solutions for $S$ then so are $y_{1}(i+\omega, j), y_{2}(i+\omega, j), \cdots, y_{L}(i+\omega, j)$.

This theorem follows from the fact that $y_{1}(i, j), y_{2}(i, j), \cdots, y_{L}(i, j)$ considered at the points of $D$ constitute $L$ sets of $L$ constants linearly independent over $D$ and that, due to the extension of each solution over $T$ described above, $y_{1}(i+\omega, j), y_{2}(1+\omega, j), \cdots, y_{L}(i+\omega, j)$ at the points of $D$ are precisely the same sets of constants as $y_{1}(i, j), y_{2}(i, j), \cdots, y_{L}(i, j)$ although the order may be different.

2. Semiperiodic solutions. We ask the question: Does there exist a solution of (1) not identically zero over $S$ and satisfying the relation

$$
y(i+\omega, j)=\rho y(i, j)
$$

where $\rho \neq 0$ is constant? We, of course, except the case where either $(i+\omega, j)$ or $(i, j)$ is a corner point of $S$ since solutions are not defined at corner points.

Let us assume a solution $y_{q}(i, j) \neq 0$ satisfying (3) and work for necessary conditions. As previously, let $y_{1}(i, j), y_{2}(i, j), \cdots, y_{1}(i, j)$ be a fundamental system of solutions for $S$. Then so are $y_{1}(i+\omega, j)$, $y_{2}(i+\omega, j), \cdots, y_{L}(i+\omega, j)$. Consequently

$$
y_{\nu}(i+\omega, j)=\sum_{\mu=1}^{L} a_{\nu \mu} y_{\mu}(i, j), \quad \quad \nu=1, \cdots, L,
$$

where $\operatorname{det}\left(a_{\nu \mu}\right) \neq 0$. Moreover

$$
y_{q}(i, j)=\sum_{\mu=1}^{L} \alpha_{\mu} y_{\mu}(i, j)
$$

where not all the $\alpha$ 's are zero. Then 


$$
\begin{aligned}
y_{q}(i+\omega, j) & =\sum_{\mu=1}^{L} \alpha_{\mu} y_{\mu}(i+\omega, j)=\sum_{\mu=1}^{L} \alpha_{\mu} \sum_{\nu=1}^{L} a_{\mu \nu} y_{\nu}(i, j) \\
& =\sum_{\nu=1}^{L}\left[\sum_{\mu=1}^{L} \alpha_{\mu} a_{\mu \nu}\right] y_{\nu}(i, j) .
\end{aligned}
$$

Also

$$
y_{q}(i+\omega, j)=\rho y_{q}(i, j)=\rho \sum_{\nu=1}^{L} \alpha_{\nu} y_{\nu}(i, j) .
$$

We can equate coefficients since $y_{1}(i, j), \cdots, y_{L}(i, j)$ are linearly independent over $D$. We get

$$
\begin{aligned}
& \left(a_{11}-\rho\right) \alpha_{1}+a_{21} \alpha_{2}+\cdots \cdots+a_{L 1} \alpha_{L}=0 \\
& a_{12} \alpha_{1}+\left(a_{22}-\rho\right) \alpha_{2}+\cdots \cdots+a_{L 2} \alpha_{L}=0 \\
& a_{1 L} \alpha_{1}+a_{2 L} \alpha_{2}+\cdots+\left(a_{L L}-\rho\right) \alpha_{L}=0 \text {. }
\end{aligned}
$$

But the $\alpha$ 's are not all zero. Hence

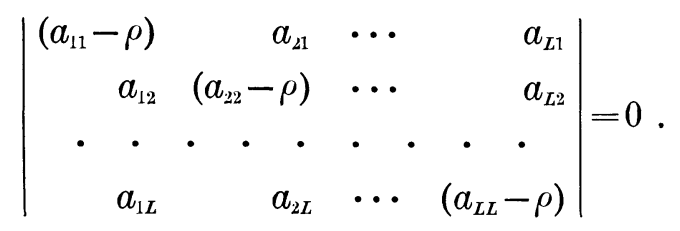

This condition is not only necessary but it is also sufficient as is seen by retracing steps.

Equation (4) is the characteristic equation for the problem and its roots are the characteristic values.

THeorem. The characteristic equation is independent of the particular fundamental system of solutions chosen.

Consider a second fundamental system, $y_{1}^{(2)}(i, j), y_{2}^{(2)}(i, j), \cdots, y_{L}^{(2)}(i, j)$. Then

$$
y_{\nu}^{(2)}(i+\omega, j)=\sum_{\mu=1}^{L} b_{\nu \mu} y_{\nu}^{(2)}(i, j), \quad \quad \nu=1, \cdots, L .
$$

The characteristic equation is

$$
\left|\begin{array}{rrrrrr}
\left(b_{11}-\rho\right) & & b_{21} & \cdots & & b_{L 1} \\
b_{12} & \left(b_{22}-\rho\right) & \cdots & & b_{L 2} \\
\cdot & \cdot & \cdot & \cdot & . & . \\
b_{1 L} & b_{2 L} & \cdots & \left(b_{L L}-\rho\right)
\end{array}\right|=0 .
$$


But

$$
y_{\nu}^{(2)}(i, j)=\sum_{\mu=1}^{L} h_{\nu \mu} y_{\mu}(i, j), \quad \quad \nu=1, \cdots, L,
$$

where $\operatorname{det}\left(h_{\nu \mu}\right) \neq 0$. Hence

$$
\begin{aligned}
y_{\nu}^{(2)}(i+\omega, j) & =\sum_{\mu=1}^{L} b_{\nu \mu} y_{\mu}^{(2)}(i, j)=\sum_{\mu=1}^{L} b_{\nu \mu} \sum_{\eta=1}^{L} h_{\mu \eta} y_{\mu}(i, j) \\
& =\sum_{\eta=1}^{L}\left[\sum_{\mu=1}^{L} b_{\nu \mu} h_{\mu \eta}\right] y_{\eta}(i, j) .
\end{aligned}
$$

On the other hand

$$
\begin{aligned}
y_{\nu}^{(2)}(i+\omega, j) & =\sum_{\mu=1}^{L} h_{\nu \mu} y_{\mu}(i+\omega, j)=\sum_{\mu=1}^{L} h_{\nu \mu} \sum_{\eta=1}^{L} a_{\mu \eta} y_{\eta}(i, j) \\
& =\sum_{\eta=1}^{L}\left[\sum_{\mu=1}^{L} h_{\nu \mu} a_{\mu \eta}\right] y_{\eta}(i, j) .
\end{aligned}
$$

We can equate coefficients, as already explained, because $y_{1}, y_{2}, \cdots, y_{L}$ are linearly independent. We have

$$
\sum_{\mu=1}^{L} b_{\nu \mu} h_{\mu \eta}=\sum_{\mu=1}^{L} h_{\nu \mu} a_{\mu \eta}, \quad \eta=1, \cdots, L ; \nu=1, \cdots, L .
$$

Now let us form the products

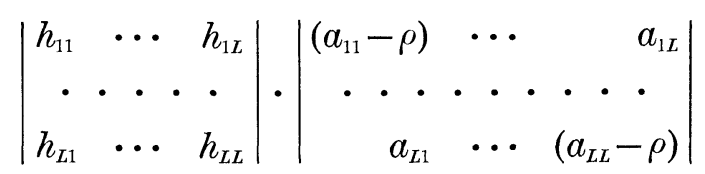

and

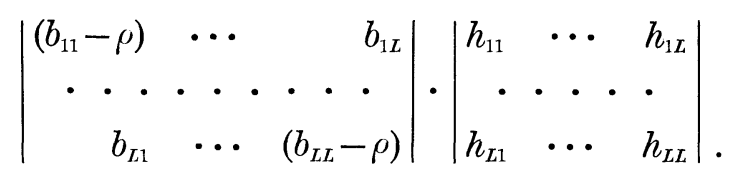

If we perform the indicated multiplication and then use (5) we get identical determinants. This establishes the theorem.

THEOREM. No characteristic value is zero.

This theorem follows from the fact that $\operatorname{det}\left(a_{i j}\right) \neq 0$.

If it were zero then $y_{1}(i+\omega, j), y_{2}(i+\omega, j), \cdots, y_{L}(i+\omega, j)$ would be linearly independent over a fundamental domain which they are not.

3. Roots distinct. Let the roots of the characteristic equation be $\rho_{1}, \rho_{2}, \cdots, \rho_{t}$ and assume that no two are equal. Let corresponding 
semiperiodic solutions be $y_{1}(i, j), \cdots, y_{L}(i, j)$, that is $y_{\nu}(i+\omega, j)=\rho_{\nu}(i, j)$, $\nu=1,2, \cdots, L$ and assume, as we can, that no one of these is identically zero.

Theorem. The solutions $y_{1}, \cdots, y_{L}$ constitute a fundamental system of solutions.

To prove this theorem we assume first $y_{1}, \cdots, y_{L-k}$ are linearly dependent over $D$ but that $y_{1}, \cdots, y_{L-k-1}$ are linearly independent over D. Then

$$
\sum_{\nu=0}^{L-k} \mu_{\nu} y_{\nu}(i, j)=0
$$

over $D$ with at least one $\mu \neq 0$. Replace $i$ by $(i+\omega)$. Then

$$
\sum_{\nu=0}^{L-k} \mu_{\nu} y(i+\omega, j)=0
$$

over $D$. This is true because solutions linearly dependent over $D$ are linearly dependent over all of $T$.

From (7)

$$
\sum_{\nu=0}^{L-k} \mu_{\nu} \rho_{\nu} y_{\nu}(i, j)=0
$$

But $\mu_{L}-k \neq 0$ else $y_{1}, \cdots, y_{L-k-1}$ would be linearly dependent. Eliminate $y_{L-k}$ between (6) and (8). We get

$$
\sum_{\nu=1}^{L-k-1} \mu_{\nu}\left(\rho_{\nu}-\rho_{L-k}\right) y_{\nu}(i, j)=0 \text {. }
$$

The only way that this can be true with the linear independence of $y_{1}, \cdots, y_{L-k-1}$ is that

$$
\mu_{1}\left(\rho_{1}-\rho_{L-k}\right)=\mu_{2}\left(\rho_{2}-\rho_{L-k}\right)=\cdots=\mu_{L-k-1}\left(\rho_{L-k-1}-\rho_{L-k}\right)=0 .
$$

If $\mu_{1}=\mu_{2}=\cdots=\mu_{L-k-1}=0$, then $\mu_{L-k} y_{L-k}(i, j) \equiv 0$. This is not the case since $\mu_{L-k} \neq 0$ and $y_{L-k}(i, j) \neq 0$ over $D$. Consequently $\rho_{L-k}$ must equal some other $\rho$. This contradicts our simple root hypothesis. Hence, $y_{1}, \cdots, y_{L}$, are linearly independent over $D$ and the theorem is proved.

4. Multiple roots; special discussion. Let us assume that $\rho_{1}$ is a double root of the characteristic equation but that all other roots are simple. Let $y_{1}(i, j)$ be as before ; namely $y_{1}(i+\omega, j)=\rho_{1} y_{1}(i, j) \neq \equiv$ and let $\tilde{y}_{2}(i, j), \tilde{y}_{3}(i, j), \cdots, \tilde{y}_{L}(i, j)$ be so chosen that $y_{1}, \tilde{y}_{2}, \tilde{y}_{3}, \cdots, \tilde{y}_{L}$ form a fundamental system. We have the relations 
THE FIVE-POINT DIFFERENCE EQUATION WITH PERIODIC COEFFICIENTS 1347

$$
\begin{aligned}
& y_{1}(i+\omega, j)=\rho_{1} y_{1}(i, j), \\
& \tilde{y}_{\nu}(i+\omega, j)=c_{\nu 1} y_{1}(i, j)+\sum_{\mu=2}^{L} c_{\nu \mu} \tilde{y}_{\mu}(i, j), \quad \nu=2, \cdots, L .
\end{aligned}
$$

The characteristic equation is

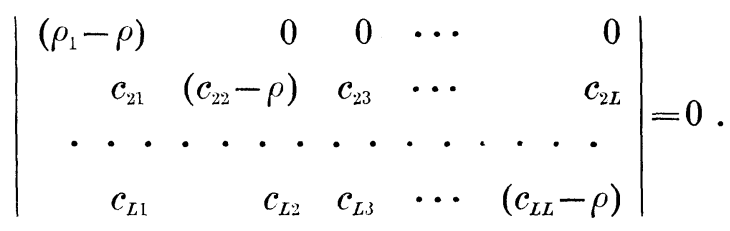

Since $\rho_{1}$ is a double root of $(10)$,

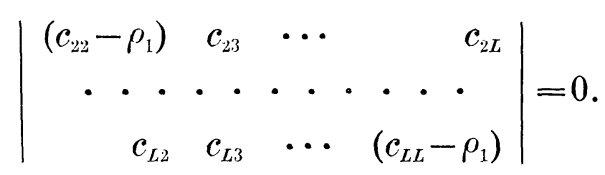

Hence from (9) the solutions $\tilde{y}_{\nu}(i+\omega, j)-c_{\nu 1} y_{1}(i, j)-\rho_{1} \tilde{y}_{\nu}(i, j), \nu=2, \cdots, L$ are linearly dependent.

This means that

$$
\sum_{\nu=2}^{L} C_{\nu} \tilde{y}_{\nu}(i+\omega, j)=y_{1}(i, j) \sum_{\nu=2}^{L} C_{\nu} c_{\nu 1}+\rho_{1} \sum_{\nu=2}^{L} C_{\nu} \tilde{y}_{\nu}(i, j) .
$$

Let $\sum_{\nu=2}^{L_{L}} C_{\nu} c_{\nu 1}=\kappa$ and $Y_{2}(i, j)=\sum_{\nu=2}^{L} C_{\nu} \tilde{y}_{\nu}(i, j)$. We note that $Y_{2}(i, j) \neq 0$ since $y_{1}, \tilde{y}_{z}, \cdots, \tilde{y}_{L}$ are linearly independent over $D$. Then

$$
Y_{2}(i,+\omega, j)=\rho_{1} Y_{2}(i, j)+\kappa y_{1}(i, j) .
$$

This is a difference equation in $Y_{2}$ as a function of $i$ with difference interval $\omega$. We shall solve ${ }^{2}$ for $Y_{2}(i+\mu \omega, j)$. Let $U(i+\mu \omega, j)$ be a solution of the difference equation

$$
U(i+\omega, j)=\rho_{1} U(i, j) .
$$

Then $U(i+\mu \omega, j)=\rho_{1}^{\mu} U(i, j)$. Moreover $U(i, j)$ is arbitrary so we assume it different from zero. Then

$$
Y_{2}(i+\mu \omega, j)=U(i+\mu \omega, j)\left[\sum_{\nu=0}^{\mu-1} \frac{\kappa y_{1}(i+\nu \omega, j)}{U(i+(\nu+1) \omega, j)}+c_{1}(i, j)\right] .
$$

We note that $y_{1}(i+\nu \omega, j)=\rho_{1}^{\nu} y_{1}(i, j)$. With this in mind (13) yields

$$
Y_{2}(i+\mu \omega, j)=\left[\frac{\kappa}{\rho_{1}} \mu y_{1}(i, j)+U(i, j) c_{1}(i, j)\right] \rho_{1}^{\mu}
$$

2 T. Fort, Finite differences and difference equations in the real domain. Clarendon, 1948 , p. 117. 
We rewrite this ${ }^{3}$

$$
Y_{2}(i+\mu \omega, j)=\left[\frac{\kappa}{\rho_{1}} \mu y_{1}(i, j)+Y_{2}(i, j)\right] \rho_{1}^{\mu} .
$$

This is an interesting form for $Y_{2}(i+\mu \omega, j)$. We note particularly the $\mu$ in the first term of the bracket.

Theorem. The solution $y_{1}(i, j), Y_{2}(i, j), y_{3}(i, j), \cdots, y_{L}(i, j)$ form a fundamental system

To prove this theorem assume the contrary, namely linear dependence :

$$
c_{1} y_{1}(i, j)+c_{2} Y_{2}(i, j)+c_{3} y_{3}(i, j)+\cdots+c_{L} y_{L}(i, j)=0 .
$$

Then increasing $i$ by $\omega$ yields

$$
c_{1} \rho_{1} y_{1}(i, j)+c_{2} \kappa y_{1}(i, j)+c_{2} \rho_{1} Y_{2}(i, j)+c_{3} \rho_{3} y_{3}(i, j)+\cdots+c_{L} \rho_{L} y_{L}(i, j)=0 .
$$

Now $c_{2}$ is not zero else $y_{1}, y_{3}, \cdots, y_{L}$ would be linearly dependent which they are not. We eliminate $Y_{2}(i, j)$ from (15) and (16). We get

$$
c_{2} \kappa y_{1}(i, j)+c_{3}\left(\rho_{3}-\rho_{1}\right) y_{3}(i, j)+\cdots+c_{L}\left(\rho_{L}-\rho_{1}\right) y_{L}(i, j)=0 \text {. }
$$

But $c_{3}, \cdots, c_{L}$ are not all zero. If they were we would have $y_{1}(i, j)$ and $Y_{2}(i, j)$ linearly dependent. They are not since $Y_{2}(i, j)$ is linearly dependent upon $\tilde{y}_{3}(i, j), \cdots, \tilde{y}_{L}(i, j)$ and by hypothesis $y_{1}(i, j)$ is not. It results that $\rho_{1}$ must equal at least one of $\rho_{3}, \cdots, \rho_{L}$. This contradicts our hypothesis.

We now assume $\rho_{1}$ a triple root but that other roots are distinct.

We consider $y_{1}(i, j)$ and $Y_{2}(i, j)$ of the double root discussion and note that they are not linearly dependent. We then define $\tilde{y}_{3}(i, j), \cdots$, $\tilde{y}_{L}(i, j)$ so that $y_{1}(i, j), Y_{2}(i, j), \tilde{y}_{3}(i, j), \cdots, \tilde{y}_{L}(i, j)$ form a fundamental system. The characteristic equation takes the form

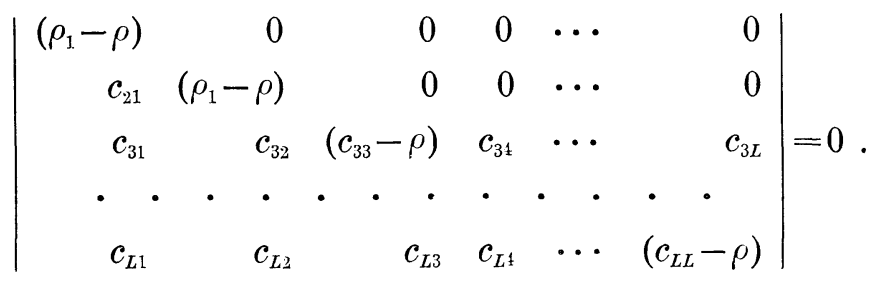

Since $\rho_{1}$ is a triple root of this equation we have

3 The convention used in (13) is $\sum_{\nu=0}^{-1} f(\nu)=0$. 


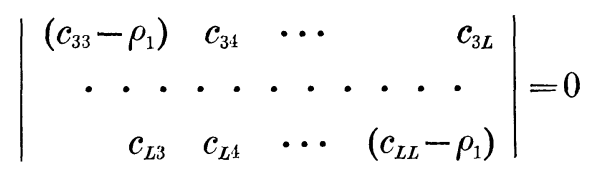

It follows from (17) that $\tilde{y}_{\nu}(i+\omega, j)-c_{\nu_{1}} y_{1}(i, j)-c_{\nu_{2}} Y_{2}(i, j)-\rho_{1} \tilde{y}_{\nu}(i, j), \nu=3$, $\cdots, L$ are linearly dependent. Let constants determining the linear dependence be $\gamma_{3}, \gamma_{4}, \cdots, \gamma_{L}$. Then let $\kappa_{\nu}=\sum_{\mu=3}^{L} \gamma_{\mu} c_{\mu \nu}, \nu=1,2$. Let

$$
Y_{3}(i, j)=\sum_{\mu=3}^{L} \gamma_{\mu} \tilde{y}_{\mu}(i, j)
$$

Then

$$
Y_{3}(i+\omega, j)=\kappa_{1} y_{1}(i, j)+\kappa_{2} Y_{2}(i, j)+\rho_{1} Y_{3}(i, j) .
$$

This is a difference equation in $Y_{3}$ as a function of $i$ with difference interval $\omega$. We solve precisely as we solved (11).

As previously we choose $U(i, j)$ not equal to zero then

$$
Y_{3}(i+\mu \omega, j)=U(i+\mu \omega, j)\left[\sum_{\nu=1}^{\mu-1} \frac{\kappa_{1} y_{1}(i+\nu \omega, j)+\kappa_{2} Y_{2}(i+\nu \omega, j)}{U(i+(\nu+1) \omega, j)}+c(i, j)\right] .
$$

Now substitute $y_{1}(i+\nu \omega, j)=\rho_{1}^{\nu} y_{1}(i, j)$ and

$$
Y_{2}(i+\nu \omega, j)=\rho_{1}^{2}\left[\frac{\kappa}{\rho_{1}^{\nu}} y_{1}(i, j)+Y_{2}(i, j)\right] .
$$

we get

$$
\begin{aligned}
Y_{3}(i+\mu \omega, j)= & \rho_{1}^{\mu-1} \mu \kappa y_{1}(i, j)+\kappa \kappa_{2} \rho_{1}^{\mu-2} \frac{\mu(\mu-1)}{3 !} y_{1}(i, j) \\
& +\kappa_{2} \rho_{1}^{\mu-1} \mu Y_{2}(i, j)+\rho_{1}^{\mu} Y_{3}(i, j)
\end{aligned}
$$

5. Multiple roots ; general discussion. The work that we have just done is easily generalized. Details are ommitted but can be readily supplied by one who has read $\S 4$.

THEOREM. If $\rho_{1}$ is an $\alpha_{1}$-fold root of the characteristic equation there exist solutions of (1) which we call $Y_{1}^{(1)}(i, j), Y_{2}^{(1)}(i, j), \cdots, Y_{\alpha_{1}}^{(1)}(i, j)$, were

$$
\begin{aligned}
& Y_{1}^{(1)}(i+\mu \omega, j)=\rho_{1}^{\mu} Y_{1}^{(1)}(i, j), \\
& Y_{2}^{(1)}(i+\mu \omega, j)=\rho_{1}^{\mu}\left[c_{1}^{(1)} \mu Y_{1}^{(1)}(i, j)+Y_{2}^{(1)}(i, j)\right], \\
& Y_{3}^{(1)}(i+\mu \omega, j)=\rho_{1}^{\mu}\left[\left\{c_{1}^{(2)} \mu+c_{2}^{(2)} \frac{\mu(\mu-1)}{2 !}\right\} Y_{1}^{(1)}(i, j)+c_{3}^{(2)} \mu Y_{2}^{(1)}(i, j)+Y_{3}(i, j)\right],
\end{aligned}
$$




$$
\begin{aligned}
& Y_{\alpha_{1}}^{(1)}(i+\mu \omega, j) \\
& =\rho_{1}^{\mu}\left[\left\{c_{1}^{\left(\alpha_{1}\right)} \mu+c_{2}^{\left(\alpha_{1}\right)} \mu(\mu-1)+\cdots+c_{\alpha_{1}-1}^{\left(\alpha_{1}\right)} \frac{\mu(\mu-1) \cdots\left(\mu-\alpha_{1}+2\right)}{\left(\alpha_{1}-1\right) !} Y_{1}^{(1)}(i, j)\right\}\right. \\
& \quad+\left\{c_{\alpha_{1}}^{\left(\alpha_{1}\right)} \mu+\cdots+c_{2 \alpha_{1}-3}^{\left(\alpha_{1}\right)} \frac{\mu(\mu-1) \cdots\left(\mu-\alpha_{1}+3\right)}{\left(\alpha_{1}-2\right) !}\right\} Y_{2}^{(1)}(i, j)+\cdots \\
& \left.\quad+c_{\left(\alpha_{1}^{2}-\alpha_{1}\right) / 2}^{\left(\alpha_{1}\right)} \mu Y_{\alpha_{1}-1}^{(1)}(i, j)+Y_{\alpha_{1}}^{(1)}(i, j)\right] .
\end{aligned}
$$

If the roots are $\rho_{1}$ of order $\alpha_{1}, \rho_{2}$ of order $\alpha_{2}, \cdots, \rho_{t}$ of order $\alpha_{t}$; then the solutions $Y_{1}^{(1)}, Y_{2}^{(1)}, \cdots, Y_{\alpha_{1}}^{(2)}, Y_{1}^{(1)}, \cdots, Y_{\alpha_{2}}^{(2)}, \cdots, Y_{1}^{(t)}, \cdots, Y_{\alpha_{t}}^{(t)}$ form a fundamental system of solutions.

UNIVERSity OF SOUTH CAROLINA 


\title{
PACIFIC JOURNAL OF MATHEMATICS
}

\author{
EDITORS
}

H. L. Royden

Stanford University

Stanford, California

R. A. Beaumont

University of Washington

Seattle 5, Washington
A. L. Whiteman

University of Southern California

Los Angeles 7, California

E. G. Straus

Unıversity of California

Los Angeles 24, California

\section{ASSOCIATE EDITORS}

\author{
E. F. BECKENBACH \\ C. E. BURGESS \\ M. HALL \\ E. HEWITT
}

\author{
A. HORN \\ V. GANAPATHY IYER \\ R. D. JAMES \\ M. S. KNEBELMAN
}

L. NACHBIN
I. NIVEN
T. G. OSTROM
M. M. SCHIFFER

G. SZEKERES

F. WOLF

K. YOSIDA

\section{SUPPORTING INSTITUTIONS}

UNIVERSITY OF BRITISH COLUMBIA

CALIFORNIA INSTITUTE OF TECHNOLOGY

UNIVERSITY OF CALIFORNIA

MON'TANA STATE UNIVERSITY

UNIVERSITY OF NEVADA

OREGON STATE COLLEGE

UNIVERSITY OF OREGON

UNIVERSITY OF SOUTHERN CALIFORNIA

\author{
STANFORD UNIVERSITY \\ UNIVERSITY OF UTAH \\ WASHINGTON STATE COLLEGE \\ UNIVERSITY OF WASHINGTON \\ AMERICAN MATHEMATICAL SOCIETY \\ CALIFORNIA RESEARCH CORPORATION \\ HUGHES AIRCRAFT COMPANY \\ THE RAMO-WOOLDRIDGE CORPORATION
}

Mathematical papers intended for publication in the Pacific Journal of Mathematics should be typewritten (double spaced), and the author should keep a complete copy. Manuscripts may be sent to any of the editors. All other communications to the editors should be addressed to the managing editor, E. G. Straus at the University of California, Los Angeles 24, California.

50 reprints per author of each article are furnished free of charge; additional copies may be obtained at cost in multiples of 50 .

The Pacific Journal of Mathematics is published quarterly, in March, June, September, and December. The price per volume (4 numbers) is $\$ 12.00$; single issues, $\$ 3.50$. Back numbers are available. Special price to individual faculty members of supporting institutions and to individual members of the American Mathematical Society: $\$ 4.00$ per volume; single issues, $\$ 1.25$.

Subscriptions, orders for back numbers, and changes of address should be sent to Pacific Journal of Mathematics, 2120 Oxford Street, Berkeley 4, California.

Printed at Kokusai Bunken Insatsusha (International Academic Printing Co., Ltd.), No. 10, 1-chome, Fujimi-cho, Chiyoda-ku, Tokyo, Japan.

PUBLISHED BY PACIFIC JOURNAL OF MATHEMATICS, A NON-PROFIT CORPORATION

The Supporting Institutions listed above contribute to the cost of publication of this Journal, but they are not owners or publishers and have no responsibility for its content or policies. 


\section{Pacific Journal of Mathematics}

\section{Vol. 7, No. $3 \quad$ March, 1957}

Silvio Aurora, Multiplicative norms for metric rings............... 1279

Ross A. Beaumont and John Richard Byrne, On the construction of

$R$-modules and rings with polynomial multiplication ............ 1305

Fred Brafman, An ultraspherical generating function . . . . . . . . . . . 1319

Howard Ernest Campbell, On the Casimir operator ............... 1325

Robert E. Edwards, Representation theorems for certain functional

operators..................................... 1333

Tomlinson Fort, The five-point difference equation with periodic

coefficients ..................................... 1341

Isidore Heller, On linear systems with integral valued solutions......... 1351

Harry Hochstadt, Addition theorems for solutions of the wave equation in

parabolic coordinates ................................ 1365

James A. Hummel, The coefficient regions of starlike functions . . . . . . . . 1381

Fulton Koehler, Estimates for the eigenvalues of infinite matrices ......... 1391

Henry Paul Kramer, Perturbation of differential operators ............. 1405

R. Sherman Lehman, Development of the mapping function at an analytic

corner .......................................... 1437

Harold Willis Milnes, Convexity of Orlicz spaces.................. 1451

Vikramaditya Singh, Interior variations and some extremal problems for certain classes of univalent functions . . . . . . . . . . . . . . . . 1485

William Lee Stamey, On generalized euclidean and non-euclidean spaces ............................................. 1505

Alexander Doniphan Wallace, Retractions in semigroups .............. 1513

R. L. Wilder, Monotone mappings of manifolds .................. 1519 